\title{
A SURVEY OF MULTISPECTRAL IMAGE DENOISING METHODS FOR SATELLITE IMAGERY APPLICATIONS
}

\author{
ANKUSH RAI*, JAGADEESH KANNAN R \\ School of Computing Science \& Engineering, VIT University, Chennai, Tamil Nadu, India. Email: ankushressci@gmail.com
}

Received: 13 December 2016, Revised and Accepted: 07 April 2017

\section{ABSTRACT}

In comparison with the standard RGB or gray-scale images, the usual multispectral images (MSI) are intended to convey high definition and an authentic representation for real world scenes to significantly enhance the performance measures of several other tasks involving with computer vision, segmentation of image, object extraction, and object tagging operations. While procuring images form satellite, the MSI are often prone to noises. Finding a good mathematical description of the learning-based denoising model is a difficult research question and many different researches accounted in the literature. Many have attempted its use with the application of neural network as a sparse learned dictionary of noisy patches. Furthermore, this approach allows several algorithm to optimize itself for the given task at hand using machine learning algorithm. However, in practices, a MSI image is always prone to corruption by various sources of noises while procuring the images. In this survey, we studied the past techniques attempted for the noise influenced MSI images. The survey presents the outline of past techniques and their respective advantages in comparison with each other.

Keywords: Multispectral image denoising, Satellite images, Learning-based denoising algorithms.

(C) 2017 The Authors. Published by Innovare Academic Sciences Pvt Ltd. This is an open access article under the CC BY license (http://creativecommons. org/licenses/by/4. 0/) DOI: http://dx.doi.org/10.22159/ajpcr.2017.v10s1.19740

\section{PROBLEM IDENTIFICATION}

The multispectral images (MSI) become ubiquitous for research advances where the scientists or professionals involving in areas like that of remote sensing, astrophotography, and geotracking activities. Equipment mainly employed for MSI imaging consists of a wide array of sensors with specific band area of light illumination. Since, such devices involve physical measurements, the recorded images get influenced or suffered from the noises or noisy signals arise during the measurement of the reflectance signals. Thus, representing the problem of noisy signals get assimilated with the non-noisy actual signal present during the recording of the imagery data can be mathematically represented in native linear algebraic form as:

$y=x+n$

Where $\mathrm{x}$ is the actual signal, $\mathrm{n}$ is the additive noise in pixel-wise form, and $\mathrm{y}$ is the recorded imagery data in pixel-wise manner at hand.

However, in the past, there are several algorithms that have been proposed to resolve the image denoising of multispectral images. This technique tends to find the approximate estimate of the noisy free signals from the recorded noisy image. Many such attempts work under a general assumption, thus leading the process to a pavement involving heavy chunk of processing with the additional requisite to manually employ thresholding techniques for denoising, which indeed is a daunting task. Further, this eliminates the mandatory elimination of some of the essential data in sacrifice to extract the noise free signal. The goal of this study is to give a survey of attempted denoising algorithm for the non-automated and automated denoising method of the MSI effectively with that of the past attempted methods cited below.

\section{INTRODUCTION}

MSI is steadily growing in popularity as a digital means for remote sensing, detection of thermal signature, and terrain analysis. It is commonly used as a feasible substitute for mapping applications when standard mapping and geodesy products are outdated or inadequate [1-5]. Thus, in the study, we summarize several denoising algorithm for the scenario of multispectral image denoising using several statistical and learning-based techniques, for which its performances are measured based on the two factors such as its computational workload and the denoised output image which readily aid the users in the process to use the well-known algorithms for detection, segmentation, and classification [6-10]. The study tends to give a very good division of the coefficients in terms of magnitude and three-phase angles to generalize better the concept of analytic signal to image promises an easy transformation for the analysis and processing of MSI with strong structural information [11,12]. Furthermore, we have also discussed the property of multispectral images such as shift invariant and directivity. The multispectral database is used for MSI image during the study $[13,14]$.

We have investigated the prior denoising methods attempted with recurrent neural networks (RNN) which was earlier proposed by Bengio $[15,16]$. This RNN approach had proved its exceptionality in various other scenarios of modeling of sequential data. The investigation of Goodman shows that the model is highly successful when a grouped model is considered that that of the single one while including the class-based model [17]. In later, studies of Schwenk have showed in his studies that the RNN-based models give desirable improvements in context of recognition systems like that of speech recognition [18]. Since, the algorithm on DPCA proves quite beneficial; thus, we implemented it with RNN to increase its effectiveness [19].

\section{TAXONOMY OF IMAGE DENOISING METHODS}

The following enlisted methods are principally implemented and widely used for various denoising applications of multispectral images.

1. General filtering techniques, which is based on general arguments and parameterization of the recorded signal.

2. Techniques based on global statistics of the image.

3. Techniques employing only internal image statistics.

4. Techniques which are based on general arguments about the recorded signal yet restricted to the internal statistics of the image.

As noted from the above methods, each of the techniques are the refinement of one or the other and few are even used in combination 
to implement over the specialized cases. Thus, in this scenario of denoising, the performance of the approach will improve when it is implemented with one or constrained over a chain of assumptions for the given multispectral imaging data, which indeed hold true; however, simultaneously, it also gives rise to a certain problem of making too many strong assumptions, therefore causing constriction or narrowing a solution too much at the level where the denoising involve the reduction of noisy signals hampering the actual signal in association. Consequently, leading to a decreased performance and increasing the computational cost of denoising. In such statistical methods, the variance versus the bias tradeoff is a common erupting problem. However, the most popular algorithm which has achieved a state of the art results mostly relied on the internal statistic of the image [20]. Hence, we note that the problem of biasing the estimation is not very big. In the following subsection, each of the method is discussed.

\section{Filtering}

The filtering-based approach for image denoising heavily relies of general arguments which vary drastically as it either holds for all sorts of MSI or in other cases even for the digital signals also. For illustration, this technique holds the assumption that the pixel values will not change form one pixel to another and thereby the neighboring pixels can be thought as similar samples to the pixels that is in the current iteration to get denoised. Algorithms for this technique include:

- Mean filtering

- Median filtering

- Bilateral filtering [21]

- Anisotropic diffusion [22].

Such methods does not require any sort of preprocessing technique for performing computational operation of denoising and thus speed up the whole process in general. Although due to its generality, the obtained results from such techniques are inferior to the task specific algorithms.

\section{Algorithms applying global image statistics}

There are also some methods which rely on global statistics of the image which also makes some general assumptions about the images. Therefore, in such types of denoising algorithm, at least some part of computation are done or the training of denoising pixels are achieved to extract the general statistics of the image. For illustration, there could have the requirement for a dictionary of clean imagery patches, and thus, the function for neighboring pixels is based on Markov random fields.

The algorithms which are based on or should we say which exploits the statistics of the natural image, i.e., its scarcity of pixels in Fourier space or on the basis of wavelet give empirically better performance since such empirical studies of the MSI require to perform computation prerequisite of formulating the denoised pattern in this approach. The methods of this approach include:

- Wavelet or dictionary-based methods which relies on decompositionbased methods, such as $[23,24]$

- Learned filter-based methods $[25,26]$.

Since in this approach, the output is already pushed toward the realistic model of the imagery data. Thus, it performs better than the previously discussed general filtering techniques with low computational time since the global statistics requires to be computed only once.

\section{Algorithms applying internal statistics}

This method heavily relies on the statistics found in the image itself. The algorithms compute on the basis of per patches which includes the classes of non-local means [27]. BM3D algorithm is one of the state of the art methods used for the application.

Its other common features include the characteristics of selfsimilarity with other sorts of natural or multispectral images, where some may argue that it is the very general idea for signal processing.
Furthermore, the BM3D algorithm is applicable for wavelet coefficient shrinking of the imager patches which is based on general statistics of the MSI. As the method works on internal statistics of the image, thus, the algorithm perform its denoising operation only over the noisy patches in the imagery data. This attempt has proven its supremacy over the other denoising approaches as it has advantage of performing no extraimage statistics during the runtime of the algorithm. Although it has longer duration of runtimes to perform local evaluation on noisy patches.

These methods have been shown to work among the best in practice and have the additional advantage that no extraimage statistics are required during runtime. However, these methods tend to have long run times since a lot of local evaluations in input images have to be performed.

\section{Combining external and internal image statistics}

In this category of denoising algorithms, the attempts have been made to compositely use the above two methods in combination with each other which comprise both internal and external statistics of the image, thus outperforming its predecessors. This has been shown in some of the few previous studies to achieve comparable results over the large number of patches related with the task of MSI denoising with super resolution $[28,29]$.

\section{Image denoising for other types of noise}

Additive white Gaussian noise (AWGN) is mostly considered in the literature of image denoising though there are also other types of noises which are also studied in our work. Although there are other types of noises which are studied in our work. Therefore, it is required for an effective algorithm that to be capable enough to denoise such various types of noises effectively in varying conditions.

\section{Mixed poisson-gaussian noise}

As we already have discussed above that the number of photons emitting from the light source is non-deterministic, but in fact distributed according to the passion distribution which in turn is proportional to the intensity of the illuminating source. Thus, in this case, the value of the signal is expected to be increased which is divided by its standard deviation, and thus, the signal to noise ratio will increase as the parameters of the poison distribution increases. Thus, in the well-illuminated environment, the source of noise introduction is the random arrival of photons and the AWGN noise dominated the deterioration of the actual signal. However, in a setting involves with the poor lighting environment, the significant amount of noise introduction to the actual signal is contributed by the stochastic nature of the signal itself, where a mixed Poisson-Gaussian distribution of noise model is taken in account upon which several denoising algorithms had been built $[30,31]$. There are also other approaches which apply suitable transformations to reduce the problem of regular AWGN noise [32].

\section{Impulse noise}

This mode of noise signals comes in various forms as they all fundamentally share different sorts of assumption regarding degradation of the actual signals as when compared to the general additive noises. In this type of noises due to the transmission pipeline or faults in the hardware of recording device, the certain percentage of pixels are completely left as void of any signal. Such noises are also categorized into salt-pepper noise and stripe noise, wherein the corrupted pixels are fixed to a specific value as these type of noises are completely different from additive noises. Therefore, a completely whole different set of approach is accompanied for the denoising process [33-36]. The trouble with this type of noises in denoising process involves with the problem that any type of filter will not work with this type of noises as the location of noisy pixel is left unknown and cannot be excluded from mean thresholding value. Hence, the algorithm prepared for such denoising is based on median filtration model with vital aspect of edge detection and corruption of the pixel. Since the recording instruments for the imagery 
signals will be influenced by Bernoulli distribution. Hence, the maximum and the minimum signals are limited to it venture. Thereby, giving surety of existing of such noises by the recording instruments.

\section{Learning-based denoising algorithm}

It is clear that there requires a certain assumption associated with the processing of the prior signal information which must be used to enable the denoising algorithm for effective operation with the prior supplement of the knowledge into it. Now, for the achievement of the same, there is prior statistical information that can be prefetched to the denoising algorithm using the variety of ways developed using the combination of both statistical information and machine learning algorithm.

Here, the use of the term prior information can be of different meaning based on its usage type. At once, it can refer to the scarcity of the wavelet coefficients and at other, it refers to the probability-based distribution for certain pixel value combination of small imagery patches. This type of approach can be used in multitude of image restoration tasks and poses a likelihood for better results.

Although finding a good mathematical description of the learning-based denoising model is a difficult research question and many different research accounted it in the literature $[25,37,38]$. Many have attempted its use with the application of neural network as a sparse-learned dictionary of noisy patches [24,26,39]. Furthermore, this approach allows several algorithm to optimize itself for the given task at hand using machine learning algorithm. Thus, same model can be used for solving different task and in different conditions [40,41]. Thats the reason why more of the studies are based on developing an automated algorithm for multispectral denoising using RNN.

\section{RESULTS AND CONCLUSIONS}

To comprehend the importance of learning-based image, denoising technique that we have portrayed in this study is to automate the denoising mechanism which involves varying parameter shift for each and different MSI reordered under different environments. In several cases, it is an enlightening fact that the signal processing for imagery signals usually involves users with an access to the various tools and denoising algorithms with the huge amount of multispectral information; where for denoising, it is dependent on assumption of parameter shift about the strength of nosie in the input data for a general model. However, for quality management of the error rectified multispectral digital imagery signals, there involves four sources of noise, such as:

1. The prime source of nosy signal is the property of light itself, i.e., in other words, the input data recorded by the array of sensors are dependent on the number of photon-emitted from an object with respect to the time, which itself is non-deterministic. Hence, causing a proportional ambiguity in changes with brightness value of each pixel position. This type of noise is also termed as AWGN.

2. The second source is the recording instrument which usually comprises the manufacturing defects and hence influencing or directly dictating the imperfection to be introduced the measurement.

3. Third, the noise generated from thermal sources adds positive variants to the actual imagery signal, which violates the zero mean approximation assumption by the AWGN model.

4. Further, it is impossible to have a same standard deviation for all the pixels in the recorded digital imagery signal which violates the assumption of a signal recorded in suitable environment will be independent of noisiness. Since such defects are well known in the field as hot pixels and dead pixels and is a defect of the recording instrument which is almost impossible to get rid of. Furthermore, the properties of the material involved in manufacturing of the components of the recording instrument get wear down to affect the purity of the signal.

Thus, in the present study, we have presented an effective comprehensive study of the previous literatures in this context of MSI denoising for various application. However, this studies lack the unsupervised learning approach to completely automate the denoising process for multiband or multispectral images, while ensuring the fact that the computational complexity of the process is kept nominal. We hope our study will aid other researchers to omnipresent look and compare their methods with the several past methods. It will simultaneously help them in finding the advantages of the previous studies and also give an ample study and opportunity to eliminate the disadvantages posed previously and subsequently to finally improve it in their denoising framework.

\section{REFERENCES}

1. Rudin LI, Osher S, Fatemi E. Nonlinear total variation based noise removal algorithms. Phys D 1992;60:1-4, 259-68.

2. Lansel S. Denoise Lab. Available from: http://www.stanford. edu/ slansel/DenoiseLab.

3. Katkovnik V, Foi A, Egiazarian K. Astola J. From local kernel to nonlocal multiple-model image denoising. Int J Comput Vis 2010;86:1-32.

4. Vansteenkiste E, Van der Weken D, Philips W, Kerre E. Perceived image quality measurement of state-of-the-art noise reduction schemes. In: Lecture Notes in Computer Science ACIVS. Vol. 4179. Antwerp, Belgium: Springer; 2006. p. 114-24.

5. Dabov K, Foi A, Katkovnik V, Egiazarian K. Color image denoising via sparse 3D collaborative filtering with grouping constraint in luminancechrominance space. In: Proceedings IEEE International Conference on Image Processing. Vol. 1. September; 2007. p. 313-6.

6. Starck J, Candes E, Donoho D. The curvelet transform for image denoising. IEEE Trans Image Process 2002;11:670-84.

7. Saghri JA, Tescher AG. Near-lossless bandwidth compression for radiometric data. Opt Eng 1991;30(7):934-9.

8. Epstein B, Hingorani R, Shapiro J, Czigler M. Multispectral KLTwavelet data compression for landsat thematic mapper images. In: Data Compression Conference, 1992. DCC '92. March; 1992. p. 200-8.

9. Tretter D, Bouman C. Optimal transforms for multispectral and multilayer image coding. IEEE Trans Image Process 1995;4(3):296-308.

10. Cagnazzo M, Poggi G, Verdoliva L. Region-based transform coding of multispectral images. IEEE Trans Image Process 2007;16(12):2916-26.

11. Foi A, Trimeche M, Katkovnik V, Egiazarian K. Practical poissoniangaussian noise modeling and fitting for single-image raw-data. IEEE Trans Image Process 2008;17(10):1737-54.

12. Foi A. Clipped noisy images: Heteroskedastic modeling and practical denoising. Signal Process 2009;89(12):2609-29.

13. Hordley S, Finalyson G, Morovic P. A multi-spectral image database and its application to image rendering across illumination, in image and graphics. In: Proceedings Third International Conference on, December; 2004. p. 394-7.

14. Finlayson G, Hordley S, Morovic P. Using the spectra cube to build a multispectral image database. In: Proceedings Second European Conference on Color in Graphics, Imaging and Vision, CGIV 2004. Aachen, Germany, April; 2004. p. 268-74.

15. Mahoney M. Text compression as a test for artificial intelligence. In: AAAI/IAAI; 1999. p. 486-502.

16. Bengio Y, Ducharme R, Vincent P. A neural probabilistic language model. J Mach Learn Res 2003;3:1137-55.

17. Schwenk H, Gauvain JL. Training neural network language models on very large corpora. In: Proceedings Joint Conference HLT/EMNLP, October; 2005

18. Scheunders P, Driesen J. Least squares interband denoising of color and multispectral images. In: Proceedings International Conference on Image Processing ICIP. Singapore 24-27 October; 2004

19. Buades Ai, Coll B, Morel JM. On Image Denoising Methods. Centre de Mathematiques et de Leurs Applications Preprint. Vol. 5. 2004.

20. Dabov K, Foi A, Katkovnik V, Egiazarian K. Image denoising by sparse 3D transform-domain collaborative filtering. IEEE Trans Image Process 2007;16(8):2080-95.

21. Tomasi C, Manduchi R. Bilateral filtering for gray and color images. In: Proceedings of the Sixth International Conference on Computer Vision (ICCV); 1998. p. 839-46.

22. Gerig G, Kubler O, Kikini R, Jolesz FA. Nonlinear anisotropic filtering of MRI data. IEEE Trans Med Imaging 1992;11(2):221-32.

23. Portilla J, Strela V, Wainwright MJ, Simoncelli EP. Image denoising using scale mixtures of Gaussians in the wavelet domain. IEEE Trans Image Process 2003;12(11):1338-51.

24. Elad M, Aharon M. Image denoising via sparse and redundant representations over learned dictionaries. Trans Image Process 
2006;15(12):736-45

25. Roth S, Black M. Fields of experts: A framework for learning image priors. In: International Conference on Computer Vision and Pattern Recognition (CVPR). Vol. 2. IEEE; 2005. p. 860-7.

26. Burger $\mathrm{H}$, Schuler $\mathrm{C}$, Harmeling S. Image denoising: Can plain neural networks compete with BM3D? In: Conference on Computer Vision and Pattern Recognition (CVPR); 2012. p. 2392-9.

27. Buades A, Coll B, Morel J. A non-local algorithm for image denoising. In: International Conference on Computer Vision and Pattern Recognition (CVPR). Vol. 2. IEEE; 2005. p. 60-5.

28. Zontak M, Irani M. Internal statistics of a single natural image. In: Computer Vision and Pattern Recognition (CVPR), IEEE Conference; 2011. p. 977-84. DOI: 10.1109/CVPR.2011.5995401.

29. Sun L, Hays J. Super-resolution from internet-scale scene matching. In: Computational Photography (ICCP), IEEE International Conference; 2012. p. 1-12. DOI: 10. 1109/ICCPhot.2012.6215221.

30. Lefkimmiatis S, Maragos P, Papandreou G. Bayesian inference on multiscale models for Poisson intensity estimation: Applications to photon-limited image denoising. IEEE Trans Image Process 2009;18(8):1724-41.

31. Luisier F, Blu T, Unser M. Image denoising in mixed Poisson-Gaussian noise. IEEE Trans Image Process 2011;20(3):696-708.

32. Mäkitalo M, Foi A. Poisson-Gaussian denoising using the exact unbiased inverse of the generalized anscombe transformation. In: International Conference on Acoustics, Speech and Signal Processing
(ICASSP). IEEE; 2012. p. 1081-4

33. Florencio DA, Schafer RW. Decision-based median filter using local signal statistics; 1994. DOI: 10.1117/12.185969.

34. Rai A. Attribute based level adaptive thresholding algorithm (ABLATA) for image compression and transmission. J Math Comput Sci 2014;12:211-8.

35. $\mathrm{Ng} \mathrm{PE}, \mathrm{Ma} \mathrm{KK}$. A switching median filter with boundary discriminative noise detection for extremely corrupted images. IEEE Trans Image Process 2006;15(6):1506-16.

36. Garnett R, Huegerich T, Chui C, He W. A universal noise removal algorithm with an impulse detector. IEEE Trans Image Process 2005;14(11):1747-54.

37. Rai A. A novel decomposable pixel component analysis algorithm for automating multispectral satellite image denoising. Res Rev J Embed Syst Appl 2015;2(3):18-25.

38. Zoran D, Weiss Y. From learning models of natural image patches to whole image restoration. In: International Conference on Computer Vision (ICCV). IEEE; 2011. p. 479-86.

39. Jain V, Seung H. Natural image denoising with convolutional networks. Adv Neural Inf Process Syst 2008;21:769-76.

40. Rai A. Dynamic data flow based spatial sorting method for GPUs: Software based autonomous parallelization. Recent Trends Parallel Comput 2014;1(1):15-8

41. Rai A. Attribute based level adaptive thresholding algorithm for object extraction. J Adv Robotics 2015;1(2):64-8. 